\title{
ON INVARIANT FINITELY ADDITIVE MEASURES FOR AUTOMORPHISM GROUPS ACTING ON TORI
}

BY

\author{
S. G. DANI
}

\begin{abstract}
Consider the natural action of a subgroup $H$ of $\operatorname{GL}(n, \mathbf{Z})$ on $\mathbf{T}^{n}$. We relate the $H$-invariant finitely additive measures on $\left(\mathbf{T}^{n}, \mathcal{L}\right)$ where $\mathcal{L}$ is the class of all Lebesgue measurable sets, to invariant subtori $C$ such that the $H$-action on either $C$ or $\mathbf{T}^{n} / C$ factors to an action of an amenable group. In particular, we conclude that if $H$ is a nonamenable group acting irreducibly on $\mathbf{T}^{n}$ then the normalised Haar measure is the only $H$-invariant finitely additive probability measure on $\left(\mathbf{T}^{n}, \mathcal{L}\right)$ such that $\mu(R)=0$, where $R$ is the (countable) subgroup consisting of all elements of finite order; this answers a question raised by $\mathrm{J}$. Rosenblatt.

Along the way we analyse $H$-invariant finitely additive measures defined for all subsets of $\mathbf{T}^{n}$ and deduce, in particular, that the Haar measure extends to an $H$-invariant finitely additive measure defined on all sets if and only if $H$ is amenable.
\end{abstract}

In [4] J. Rosenblatt characterises the normalised Haar measure on $\mathbf{T}^{n}$ (the $n$ dimensional torus), where $n \geq 2$, as the only finitely additive probability measure defined on the class of all Lebesgue measurable sets which is absolutely continuous with respect to the Haar measure and invariant under the usual action of $\operatorname{SL}(n, \mathbf{Z})$ as a group of automorphisms of $\mathbf{T}^{n}$. This suggests two questions: (i) Is the absolute continuity condition necessary in the characterisation? (ii) Which proper subgroups of $\operatorname{SL}(n, \mathbf{Z})$ could be substituted for $\operatorname{SL}(n, \mathbf{Z})$ in the above assertion? The first question was raised in $[4]$ in the following specific form: Is it possible to replace the absolute continuity condition by the condition that the subgroup $R$ of $\mathbf{T}^{n}$, consisting of elements of finite order, have zero measure? The second question is motivated by the fact that some examples of such subgroups are implicit in the arguments in [4]; e.g. a free nonabelian subgroup acting freely on nontrivial characters under the adjoint action, etc. In this note we obtain a complete answer to both questions in a combined form (see Theorems 1 and 4 below).

A related problem is to describe, given a subgroup $H$ of the automorphism group of $\mathbf{T}^{n}$, all $H$-invariant finitely additive probability measures $\mu$ defined on $\left(\mathbf{T}^{n}, \mathcal{P}\left(\mathbf{T}^{n}\right)\right.$ ), where $\mathcal{P}\left(\mathbf{T}^{n}\right)$ is the class of all subsets of $\mathbf{T}^{n}$. It is well known that given an action of an amenable group there exist invariant finitely additive probability measures defined on the class of all subsets (cf. [3, Theorem 5.1]). Our results show that for an automorphism group $H$ of $\mathbf{T}^{n}$ any $\mu$ as above arises from an $H$-invariant subtorus $C$ on which the $H$-action factors to an action of an amenable group; $\mu$ is then concentrated on the set $\left\{x \in \mathbf{T}^{n} \mid x^{r} \in C\right.$ for some $\left.r\right\}$ (cf. Theorem 2.4). A particular consequence of this is that the Haar measure on $\mathbf{T}^{n}$ extends

Received by the editors November 9, 1983 and, in revised form, January 4, 1984.

1980 Mathematics Subject Classification. Primary 28A70; Secondary 43A07.

Key words and phrases. Invariant finitely additive measures, invariant means.

(C) 1985 American Mathematical Society $0002-9947 / 85 \$ 1.00+\$ .25$ per page 
to an $H$-invariant finitely additive measure defined on all sets if and only if $H$ is amenable (cf. Corollary 2.6). It may be noted that an analogous result holds for subgroups of the orthogonal group $O(n)$ acting on $S^{n-1}$ (cf. [6, Theorem 5]).

Let $\mathbf{T}^{n}$ be the $n$-dimensional torus where $n \geq 2, \mathcal{L}$ the $\sigma$-algebra of all Lebesgue measurable sets, and $m$ the normalised Haar measure on $\left(\mathbf{T}^{n}, \mathcal{L}\right)$. We consider the action of $\operatorname{GL}(n, \mathbf{Z})$, the group of integral $n \times n$ matrices of determinant \pm 1 , as the group of all (continuous) automorphisms of $\mathbf{T}^{n}$. Let $H$ be a subgroup of GL $(n, \mathbf{Z})$. Let $C$ be a subgroup of $\mathbf{T}^{n}$ invariant under the $H$-action (obtained by restriction). We denote by $H(C)$ the group of all automorphisms of $C$ obtained by restriction of actions of elements of $H$ to $C$. Also, given an $H$-invariant closed subgroup $C$, the $H$-action of $\mathbf{T}^{n}$ factors to an $H$-action on the torus $\mathbf{T}^{n} / C$, again as a group of automorphisms. We denote by $H\left(\mathbf{T}^{n} / C\right)$ the group of automorphisms of $\mathbf{T}^{n} / C$ which are factors of elements of $H$. We note that $H(C)$ and $H\left(\mathbf{T}^{n} / C\right)$ are factor groups of $H$ by subgroups consisting of elements whose induced action (on $C$ or $\mathbf{T}^{n} / C$, respectively) is trivial. Our main result can be stated as follows:

1. TheOREM. Let $H$ be a subgroup of $\operatorname{GL}(n, \mathbf{Z})$. Suppose there exists an $H$ invariant finitely additive probability measure $\mu$ on $\mathbf{T}^{n}$ defined for all Lebesgue measurable sets and such that $\mu \neq m$. Then there exists an $H$-invariant proper subtorus (closed connected subgroup) $C$ of $\mathbf{T}^{n}$ such that at least one of the following conditions holds: either

(i) $H\left(\mathbf{T}^{n} / C\right)$ is amenable, or

(ii) $H(C)$ is amenable and $\mu(R(C))>0$ where

$$
R(C)=\left\{x \in \mathbf{T}^{n} \mid x^{r} \in C \text { for some } r\right\} .
$$

The action of a subgroup $H$ of $\operatorname{GL}(n, \mathbf{Z})$ on $\mathbf{T}^{n}$ is said to be irreducible if there does not exist any proper nontrivial subtorus which is $H$-invariant. Following are some examples of subgroups acting irreducibly: (a) $\operatorname{SL}(n, \mathbf{Z})$, (b) $\operatorname{Sp}(n, \mathbf{Z})$, the subgroup consisting of symplectic matrices with integral entries, where $n$ is even, (c) $\mathrm{SO}(Q)_{\mathbf{z}}$, where $Q$ is an indefinite quadratic form with integral coefficients and $\mathrm{SO}(Q)_{\mathbf{z}}$ is the subgroup of $\operatorname{SL}(n, \mathbf{Z})$ of elements leaving $Q$ invariant. (d) More generally if $G$ is a semisimple algebraic subgroup of $\operatorname{GL}(n, \mathbf{C})$ defined over $\mathbf{Q}$ and acting irreducibly on $\mathbf{C}^{n}$ then $G \cap \operatorname{SL}(n, \mathbf{Z})$ acts irreducibly on $\mathbf{T}^{n}$. We also note that all of these subgroups are nonamenable. For such subgroups we have the following.

2. COROLlary. Let $H$ be a nonamenable subgroup of $\mathrm{GL}(n, \mathbf{Z})$ acting irreducibly on $\mathbf{T}^{n}$. Then there does not exist any $H$-invariant finitely additive probability measure on $\left(\mathbf{T}^{n}, \mathcal{L}\right)$ other than $m$ for which $\mu(R)=0$, where $R$ is the subgroup consisting of elements of finite order.

The corollary, in particular, answers affirmatively the question raised by Rosenblatt (in the stronger form; cf. [4, p. 634]).

3. COROLlaRY. Let $H$ be a nonamenable subgroup of $\operatorname{GL}(n, \mathbf{Z})$ such that the adjoint action on $\mathbf{Z}^{n}$ of the $H$-action on $\mathbf{T}^{n}$ is free on $\mathbf{Z}^{n}-(0)$. Let $\mu$ be an $H$ invariant finitely additive measure on $\left(\mathbf{T}^{n}, \mathcal{L}\right)$. Then either $\mu=m$ or there exists an $H$-invariant proper subtorus $C$ such that $\mu(R(C))>0$, where $R(C)$ is as in Theorem 1. 
In $\S 5$ we shall also give an action of $\operatorname{SL}(2, \mathbf{Z})$ on $\mathbf{T}^{n}$, for any $n \geq 2$, such that $m$ is the only invariant finitely additive probability measure $\mu$ for which $\mu(R)=0$, with $R$ as in Corollary 2 .

We also prove the following theorem which shows that situations as in conditions (i) or (ii) indeed give rise to measures other than $m$.

4. THEOREM. Let $H$ be a subgroup of $\mathrm{GL}(n, \mathbf{Z})$ and let $C$ be any proper closed $H$-invariant subgroup of $\mathbf{T}^{n}$. Then:

(i) If $H\left(\mathbf{T}^{n} / C\right)$ is amenable there exists an $H$-invariant finitely additive probability measure $\mu$ on $\left(\mathbf{T}^{n}, \mathcal{L}\right)$ such that $\mu \neq m$, but $\mu$ is absolutely continuous with respect to $m$.

(ii) If $H(C)$ is amenable there exists an $H$-invariant finitely additive probability measure $\mu$ such that $\mu(R(C))=1$ and $\mu(R(D))=0$ for any closed subgroup $D$ of smaller dimension than $C$.

In view of the role played by the notion of amenability in these questions, it may be worthwhile to recall here a result of J. Tits [5] that any subgroup of $\operatorname{GL}(n, \mathbf{C})$ either contains a free nonabelian subgroup or contains a solvable subgroup of finite index; it is amenable if and only if the latter holds. We do not, however, need this characterization in any of the proofs.

Since the present paper was written the author has been able to prove an analogue of Theorem 2.4 for a large class of group actions, including the actions of subgroups of the isometry groups of spheres; in particular, the conjecture on p. 81 in [6] is proved. That method however does not apply to the case at hand. The details will appear elsewhere.

ACKNowledgement. The author would like to thank S. Wagon, J. Mycielski and the referee for useful suggestions and the Centre for Advanced Study in Mathematics, Chandigarh, for their hospitality while this work was done.

1. Isotropy subgroups of actions of $\mathbf{T}^{n}$ and $\mathbf{Z}^{n}$. Let $\mathbf{C}^{n}$ and $\mathbf{R}^{n}$ be the spaces of $n$-rowed column vectors with complex and real entries respectively. We have two actions of $\operatorname{GL}(n, \mathbf{C})$, the general linear group on $\mathbf{C}^{n}$ : the natural action by left multiplication and the contragradient action under which the action of $A \in \mathrm{GL}(n, \mathbf{C})$ consists of left multiplication by ${ }^{\mathrm{t}} A^{-1}$ (where ${ }^{\mathrm{t}}$ denotes 'transpose'). These we denote by $\rho$ and $\sigma$ respectively. We note that both these are algebraic actions of the algebraic group $\operatorname{GL}(n, \mathbf{C})$. A related simple observation that we need is that the isotropy subgroup of any $z \in \mathbf{C}^{n}$ under either of the actions is an algebraic subgroup of $\mathrm{GL}(n, \mathbf{C})$.

The actions $\rho$ and $\sigma$ restrict to actions of $\operatorname{GL}(n, \mathbf{R})$ on $\mathbf{R}^{n}$; we shall denote restrictions by the same symbol as the original. Let $\mathbf{Z}^{n}$ be the subgroup consisting of the column vectors with integral entries. The subgroup $\operatorname{GL}(n, \mathbf{Z})$, consisting of integral matrices with determinant \pm 1 , leaves $\mathbf{Z}^{n}$ invariant under either of the actions.

Let $\mathbf{T}^{n}=\mathbf{R}^{n} / \mathbf{Z}^{n}$. On $\mathbf{T}^{n}$ we shall invariably have the action of $\operatorname{GL}(n, \mathbf{Z})$ obtained as the factor of the natural action $\rho$; i.e. $A\left(v+\mathbf{Z}^{n}\right)=A v+\mathbf{Z}^{n}$ for all $v \in \mathbf{R}^{n}$ and $A \in \mathrm{GL}(n, \mathbf{Z})$. On the other hand, unless stated otherwise by the $\mathrm{GL}(n, \mathbf{Z})$-action on $\mathbf{Z}^{n}$, we shall always mean the restriction of the contragradient action $\sigma$. 
Let $X$ be the character group of $\mathbf{T}^{n}$. Then the $\operatorname{GL}(n, \mathbf{Z})$-action on $\mathbf{T}^{n}$ induces an adjoint (left) action of $\operatorname{GL}(n, \mathbf{Z})$ on $X$ given by the formula $A(\gamma)(x)=\gamma\left(A^{-1}(x)\right)$ for all $x \in \mathbf{T}^{n} . \gamma \in X$ and $A \in \mathrm{GL}(n, \mathbf{Z})$. The character group $X$ is isomorphic to $\mathbf{Z}^{n}$ (which we view as $n$-rowed integral column vectors) and, for an appropriate (canonical) choice of the isomorphism, the adjoint action of $\mathrm{GL}(n, \mathbf{Z})$ on $X$ corresponds to the contragradient action on $\mathbf{Z}^{n}$ described above. This is the reason for the convention adopted above.

In the sequel, for any subgroup $S$ of $\operatorname{GL}(n, \mathbf{Z})$ and $x \in \mathbf{T}^{n}$ or $\mathbf{Z}^{n}$, we shall denote by $S_{x}$ the isotropy (stabiliser) subgroup of the $S$-action obtained by restriction of the $\mathrm{GL}(n . \mathbf{Z})$-action on the respective space as specified earlier.

Given a nonamenable subgroup $H$ of $\operatorname{GL}(n . \mathbf{Z})$ we would like to isolate a class of subgroups of $H$ which are 'minimal' nonamenable in a certain sense--see the class $\mathcal{M}(H)$ defined below. Let $H$ be a nonamenable subgroup of $\operatorname{GL}(n, \mathbf{Z})$; this we fix through $\S 1$. For any subgroup $S$ of $\mathrm{GL}(n, \mathbf{Z})$ let $Z(S)$ denote the smallest algebraic subgroup of $\mathrm{GL}(n, \mathbf{C})$ containing $S$ (viz. the Zariski closure of $S)$. Put

$$
\mathcal{N}(H)=\{Z(S) \mid S \text { a nonamenable subgroup of } H\} .
$$

Being a family of algebraic subgroups, $\mathcal{N}(H)$ admits minimal elements; any element of $\mathcal{N}(H)$ contains a minimal element. Put

$$
\mathcal{M}(H)=\{S \mid S \text { a nonamenable subgroup of } H
$$

such that $Z(S)$ is minimal in $\mathcal{N}(H)\}$.

1.2 Lemma. Let $S \in \mathcal{M}(H)$. Then under the $S$-action on $\mathbf{C}^{n}$ (obtained by restricting $\rho$ or $\sigma)$ the isotropy subgroup of any $x \in \mathbf{C}^{n}$ is either amenable or the whole of $S$.

PROOF. Let $S_{r}$ be the isotropy subgroup of $x$ under the $S$-action in question. Since the isotropy subgroup under the corresponding $\operatorname{GL}(n, \mathbf{C})$-action is algebraic, it follows that $S_{x}=S \cap Z\left(S_{x}\right)$. If $S_{x}$ is not amenable, by minimality of $Z(S)$ we have $Z\left(S_{x}\right)=Z(S)$. Hence $S_{x}=S \cap Z\left(S_{x}\right)=S \cap Z(S)=S$.

1.3 Lemma. Let $S \in \mathcal{M}(H)$ and consider the $S$-action on $\mathbf{T}^{n}$ obtained as the restriction of the (natural) $\mathrm{GL}(n, \mathbf{Z})$-action. Let $x \in \mathbf{T}^{n}$. Then either the isotropy subgroup of $x$ is amenable or there exists $r \in \mathbf{N}$ such that $x^{r}$ is fixed by $S$.

ProOf. Let $C_{x}$ denote the closed subgroup of $\mathbf{T}^{n}$ generated by $x$ and let $C_{x}^{0}$ be the comnected component of the identity in $C_{x}$. Then $C_{x}^{0}$ is of finite index in $C_{r}$. say $r$. Put $y=x^{r}$. Since $S$ acts as group automorphisms, $S_{x} \subset S_{y}$. Since any subgroup of an amenable group is amenable, it is enough to prove that either $S_{y}=S$ or $S_{y}$ is amenable. But $A \in S_{y}$ if and only if $A$ fixes each element of the subtorus $C_{r}^{0}$. Also $A \in \operatorname{GL}(n, \mathbf{Z})$ fixes each element of a subtorus $D$ if and only if under the (natural) action $\rho$ on $\mathbf{R}^{n}, A$ fixes each element of the unique subspace II of $\mathbf{R}^{\prime \prime}$ such that $W+\mathbf{Z}^{n}=D$. Combining these observations we deduce that $S_{y}=S \cap Z$ for some algebraic subgroup $Z$ of $\left(\mathrm{xL}(n, \mathbf{C})\right.$. But then $S_{y}=S \cap Z\left(S_{y}\right)$. The argument (an now be completed as in Lemma 1.2.

In the secuel. for any subgroup $Q$ of $\operatorname{GL}(n, \mathbf{Z})$ we put

$$
\begin{aligned}
& E(Q)=\left\{x \in \mathbf{Z}^{n} \mid Q \text { fixes } x\right\}, \\
& F(Q)=\left\{x \in \mathbf{T}^{n} \mid Q \text { fixes } x^{r} \text { for some } r\right\} .
\end{aligned}
$$


Each $E(Q)$ is the intersection with $\mathbf{Z}^{n}$ of a subspace in $\mathbf{R}^{n}$. Hence the intersection of $E\left(Q_{\alpha}\right)$, where $\left\{Q_{\alpha}\right\}$ is any family of subgroups of $\operatorname{GL}(n, \mathbf{Z})$, coincides with the intersection of suitably chosen finitely many $E\left(Q_{\alpha}\right)$ 's. We now prove a similar result for $F(Q)$ 's. Each $F(Q)$ belongs to the class $\mathcal{F}$ of subgroups of $\mathbf{T}^{n}$ defined as follows. For each subtorus (closed connected subgroup) $D$ of $\mathbf{T}^{n}$ let

$$
R(D)=\left\{x \in \mathbf{T}^{n} \mid x^{r} \in D \text { for some } r \in \mathbf{N}\right\}
$$

and

$$
\mathcal{F}=\left\{R(D) \mid D \text { a subtorus of } \mathbf{T}^{n}\right\} .
$$

1.6 LEMmA. If $D_{1}$ and $D_{2}$ are subtori of $\mathbf{T}^{n}$ then $R\left(D_{1}\right) \cap R\left(D_{2}\right)=R(D)$, where $D$ is the connected component of the identity in $D_{1} \cap D_{2}$. If $\left\{D_{\alpha}\right\}_{\alpha \in \Lambda}$ is a family of subtori then there exist finitely many $\alpha_{1}, \ldots, \alpha_{k} \in \Lambda$ such that

$$
\bigcap_{\alpha \in \Lambda} R\left(D_{\alpha}\right)=\bigcap_{i=1}^{k} R\left(D_{\alpha_{i}}\right) .
$$

Thus $\mp$ is closed under intersections and intersection of any subclass of elements of $₹$ equals a finite intersection.

Proof. Let $D_{1}, D_{2}$ be two subtori and let $x \in R\left(D_{1}\right) \cap R\left(D_{2}\right)$. Then there exist $r$ and $s$ such that $x^{r} \in D_{1}$ and $x^{s} \in D_{2}$. Then $x^{r s} \in D_{1} \cap D_{2}$. The connected component $D$ of $D_{1} \cap D_{2}$ (which may not be connected) has finite index, say $q$, in $D_{1} \cap D_{2}$. Hence $x^{q r s} \in D$ so that $x \in R(D)$. Thus $R\left(D_{1}\right) \cap R\left(D_{2}\right) \subset R(D)$. The other way inclusion is obvious. By induction we conclude that intersection of any finitely many elements of $\mathcal{F}$ is again in $\mathcal{F}$. Considerations of dimension of the connected component then show that intersection of $R\left(D_{\alpha}\right)$, where $\left\{D_{\alpha}\right\}$ is a family of subtori, must equal $R\left(D_{\alpha_{1}}\right) \cap R\left(D_{\alpha_{2}}\right) \cap \cdots \cap R\left(D_{\alpha_{k}}\right)$ for suitably chosen elements $\alpha_{1}, \alpha_{2}, \ldots, \alpha_{k}$.

2. Universally defined invariant measures. In this section we study $H$ invariant finitely additive measures defined for all subsets of $\mathbf{T}^{n}$. For any set $E$ we denote by $\mathcal{P}(E)$ the class of all subsets of $E$, and by $B(E)$ the space of all bounded complex-valued functions on $E$. If $\mu$ is a finitely additive probability measure on $(E, P(E))$ then we get a mean $\varphi_{\mu}$ on $B(E)$ by putting $\varphi_{\mu}(f)=\int f d \mu$ for all $f \in B(E)$ (cf. [1] for the definition of the integral). We recall the following lemma from [4] (cf. [4, Proposition 3.5]).

2.1 LEMMA. Let $G$ be a group acting on a set $E$ and suppose there exists a $G$ invariant mean on $B(E)$. Suppose also that the isotropy subgroup of each element of $E$ is amenable. Then $G$ is amenable.

In the sequel, if $\varphi$ is a mean on $B(E)$ then $\varphi(A)$, where $A$ is a subset of $E$, denotes $\varphi\left(\chi_{A}\right)$, where $\chi_{A}$ is the characteristic function of $A$ on $E$. Also let other notations, viz. $\mathcal{M}(H), E(Q), F(Q), R(C)$, etc. be as in $\S 1$. From Lemma 2.1 we deduce the following.

2.2 Proposition. Let $H$ be a nonamenable subgroup of $\operatorname{GL}(n, \mathbf{Z})$. Let $N$ be the subgroup generated by $\{S \in \mathcal{M}(H)\}$. Let $X=\mathbf{T}^{n}$ or $\mathbf{Z}^{n}$ and consider the $H$-action on $X$ obtained by restriction of the respective $\mathrm{GL}(n, \mathbf{Z})$-action (specified 
earlier). Let $\varphi$ be an $H$-invariant mean on $B(X)$. Then $\varphi(F(N))=1$ if $X=\mathbf{T}^{n}$ and $\varphi(E(N))=1$ if $X=\mathbf{Z}^{n}$.

PROOF. We give the proof for $X=\mathbf{T}^{n}$. The proof for the other case is similar. We first claim that for any $S \in \mathcal{M}(H), \varphi(X-F(S))=0$. Suppose otherwise; then the map $f \mapsto \varphi(f) / \varphi(X-F(S))$ for all $f \in B(X-F(S))$ defines an $S$ invariant mean on $B(X-F(S))$. Also by Lemma 1.3 the isotropy subgroup of any $x \in X-F(S)$ under the $S$-action is amenable. By Lemma 2.1 these two assertions imply that $S$ is amenable which is a contradiction. Hence $\varphi(X-F(S))=0$ for all $S \in \mathcal{M}(H)$. Since $N$ is generated by $\{S \in \mathcal{M}(H)\}$, we obtain $F(N)=\bigcap\{F(S) \mid S \in$ $\mathcal{M}(H)\}$. By Lemma 1.6 there exist finitely many $S_{1}, S_{2}, \ldots, S_{k} \in \mathcal{M}(H)$, such that $F(N)=F\left(S_{1}\right) \cap F\left(S_{2}\right) \cap \cdots \cap F\left(S_{k}\right)$. Hence,

$$
\varphi(X-F(N))=\varphi\left(\bigcup_{1}^{k}\left(X-F\left(S_{i}\right)\right)\right) \leq \sum_{1}^{k} \varphi\left(X-F\left(S_{i}\right)\right)=0 .
$$

Hence. $\varphi(F(N))=1$.

2.3 (OROLlary. Let $H$ be a nonamenable subgroup of $\mathrm{GL}(n, \mathbf{Z})$ and let $\mu$ be an $H$-invariant finitely additive probability measure on $\left(\mathbf{T}^{n}, \mathcal{P}\left(\mathbf{T}^{n}\right)\right)$. Then there exists an $H$-invariant proper subtorus $C$ of $\mathbf{T}^{n}$ such that $\mu(R(C))=1$.

PROOF. Let $\varphi=\varphi_{\mu}$ be the mean on $B\left(\mathbf{T}^{n}\right)$ corresponding to $\mu$. Since $H$ is not amenable, the subgroup $N$ generated by $\{S \in \mathcal{M}(H)\}$ is a nontrivial normal subgroup of $H$. But, by Proposition 2.2, $\varphi(F(N))=1$, that is, $\mu(F(N))=1$. But, as noted earlier, $F(N)=R(C)$, where $C$ is the connected component of the subgroup of elements of $\mathbf{T}^{n}$ which are pointwise fixed by $N$. Since $N$ is nontrivial, $C$ is proper. and since $N$ is normal, $C$ is $H$-invariant.

2.4 TheOREM. Let $H$ be a subgroup of $\operatorname{GL}(n, \mathbf{Z})$ and let $\mu$ be an $H$-invariant finitely additive probability measure on $\left(\mathbf{T}^{n}, \mathcal{P}\left(\mathbf{T}^{n}\right)\right)$. Then there exists an $H$ invariant subtorus $C$ of $\mathbf{T}^{n}$ such that $H(C)$ is amenable and $\mu(R(C))=1$.

PROOF. If $H$ is amenable we may choose $C=\mathbf{T}^{n}$. Now suppose $H$ is nonamenable. By Corollary 2.3 there exist proper $H$-invariant subtori $D$ such that $\mu(R(D))=1$. Let $C$ be a proper $H$-invariant subtorus of minimum possible dimension such that $\mu(R(C))=1$. We prove that $H(C)$ is amenable.

For cach $x \in R(C)$ let $r(x)=\min \left\{j \in \mathbf{N} \mid x^{j} \in C\right\}$. For each subset $A$ of $C$ put

$$
\mu^{\prime}(A)=\mu\left(\left\{x \in R(C) \mid x^{r(x)} \in A\right\}\right) .
$$

It is casy to see that $\mu^{\prime}$ is an $H(C)$-invariant finitely additive probability measure on $C$. Suppose $H(C)$ is not amenable. Then applying Corollary 2.3 to the measure $\mu^{\prime}$ on $C$ (defined in fact for all subsets), we deduce that there exists a proper $H$-invariant subtorus $D$ of $C$ such that

$$
\mu^{\prime}\left(\left\{x \in C \mid x^{j} \in D \text { for some } j\right\}\right)=1 .
$$

In other words $\mu(R(D))=1$; but this contradicts the choice of $C$. Hence $H(C)$ is amenable. 
2.5 CoRollary. Let $H$ be a nonamenable subgroup of $\operatorname{GL}(n, \mathbf{Z})$ and let $\mu$ be an $H$-invariant finitely additive measure on $\left(\mathbf{T}^{n}, \mathcal{L}\right)$ (where $\mathcal{L}$ is the class of all Lebesgue measurable subsets of $\left.\mathbf{T}^{n}\right)$. Suppose $\mu$ is not absolutely continuous with respect to $m$. Then there exists a proper $H$-invariant subtorus $C$ of $\mathbf{T}^{n}$ such that $H(C)$ is amenable and $\mu(R(C))>0$.

PROOF. Since $\mu$ is not absolutely continuous with respect to $m$ there exists $E \in \mathcal{L}$ such that $m(E)=0$ and $\mu(E)>0$. Replacing $E$ by $H E$ if necessary, we may assume $E$ to be $H$-invariant. Since $m(E)=0$, all subsets of $E$ are Lebesgue measurable and $\mu$ is defined for them. Putting $\nu(A)=\mu(A \cap E) / \mu(E)$ for all $A \in$ $\mathcal{P}\left(\mathbf{T}^{n}\right)$ defines an $H$-invariant finitely additive probability measure on $\left(\mathbf{T}^{n}, \mathcal{P}\left(\mathbf{T}^{n}\right)\right)$. Hence by Theorem 2.4 there exists a proper $H$-invariant subtorus $C$ such that $H(C)$ is amenable and $\nu(R(C))=1$. Then $\mu(R(C)) \geq \mu(R(C) \cap E)=\mu(E)>0$.

2.6 COROLlaRY. Let $H$ be a subgroup of $\mathrm{GL}(n, \mathbf{Z})$. Let $\lambda$ be an $H$-invariant countably additive probability measure on $\left(\mathbf{T}^{n}, \mathcal{L}\right)$ absolutely continuous with respect to $m$ (including possibly $\lambda=m$ ). Then there exists an $H$-invariant finitely additive measure $\mu$ on $\left(\mathbf{T}^{n}, \mathcal{P}\left(\mathbf{T}^{n}\right)\right)$ such that $\mu(A)=\lambda(A)$ for all $A \in \mathcal{L}$ if and only if $H$ is amenable.

ProOF. If $H$ is amenable then by a well known theorem (cf. [3, Theorem 5.1]) there exists an $H$-invariant finitely additive measure $\mu$ extending $\lambda$; that is, such that $\mu(A)=\lambda(A)$ for all $A \in \mathcal{L}$. Conversely, let $\mu$ be an $H$-invariant finitely additive probability measure on $\left(\mathbf{T}^{n}, \mathcal{P}\left(\mathbf{T}^{n}\right)\right)$ extending $\lambda$. By Theorem 2.4 there exists an $H$-invariant subtorus $C$ such that $H(C)$ is amenable and $\mu(R(C))=1$. Since $\mu$ extends $\lambda$ we have $\lambda(R(C))=1$, which implies $C=\mathbf{T}^{n}$. Hence $H=H\left(\mathbf{T}^{n}\right)$ is amenable.

We next prove the following converse of Theorem 2.4 (and Corollary 2.5); it covers Theorem 4 under condition (ii).

2.7 TheOREM. Let $H$ be a subgroup of $\operatorname{GL}(n, \mathbf{Z})$. Let $C$ be an $H$-invariant subtorus of $\mathbf{T}^{n}$ such that $H(C)$ is amenable. Then there exists an $H$-invariant finitely additive probability measure $\mu$ on $\left(\mathbf{T}^{n}, \mathcal{P}\left(\mathbf{T}^{n}\right)\right)$ such that

(i) $\mu(R(C))=1$,

(ii) $\mu(R(D))=0$ for any proper $H$-invariant subtorus $D$ of $C$ and,

(iii) if $C \neq \mathbf{T}^{n}$, for any $s \in \mathbf{N}, \mu\left(\left\{x \in \mathbf{T}^{n} \mid x^{j} \in C\right.\right.$ for some $\left.\left.j \leq s\right\}\right)=0$.

Proof. For each $s \in \mathbf{N}$ let $C_{s}$ be the subgroup of $\mathbf{T}^{n}$ such that $C \subset C_{s} \subset R(C)$, and $C_{s} / C$ is the subgroup of $R(C) / C$ generated by all elements of order $\leq s$. Then each $C_{s}$ is $H$-invariant and $R(C)=\bigcup C_{s}$. It is straightforward to verify that for any $s$ the restriction homomorphism $\beta: H\left(C_{s}\right) \rightarrow H(C)\left(\beta(A)\right.$ for any $A \in H\left(C_{s}\right)$ is the restriction of $A$ to $C$ ) has finite kernel. Since $\beta$ is surjective and $H(C)$ is amenable this implies $H\left(C_{s}\right)$ is amenable for any $s$ (cf. [1] ). Hence by a result of $\mathrm{J}$. Mycielski (cf. [3, Theorems 5.1 and 6.1]) for each $s$ there exists an $H\left(C_{s}\right)$-invariant finitely additive probability measure $\nu_{s}$ on $\left(C_{s}, \mathcal{P}\left(C_{s}\right)\right)$ such that $\nu_{s}(A)=0$ for any set which is of first category in $C_{s}$, and $\nu_{s}(x C)=N_{s}^{-1}$ for all $x \in C_{s}$, where $N_{s}$ is the index of $C$ in $C_{s}$. For each $s$ let $\mu_{s}$ be the finitely additive probability measure on $\left(\mathbf{T}^{n}, \mathcal{P}\left(\mathbf{T}^{n}\right)\right)$ defined by $\mu_{s}(E)=\nu_{s}\left(E \cap C_{s}\right)$ for all $E \in \mathcal{P}\left(\mathbf{T}^{n}\right)$. Then each $\mu_{s}$ is $H$-invariant. Now let $\varphi_{s}$ be the mean on $B\left(\mathbf{T}^{n}\right)$ corresponding to $\mu_{s}$, that 
is, $\varphi_{s}(f)=\int f d \mu_{s}$. The space of means is compact with respect to the weak ${ }^{*}-$ topology (cf. $[\mathbf{1}])$. Let $\varphi$ be a limit point of $\left\{\varphi_{s}\right\}$. Then $\varphi$ is an $H$-invariant mean on $B\left(\mathbf{T}^{n}\right)$. Let $\mu$ be the finitely additive probability measure on $\left(\mathbf{T}^{n}, \mathcal{P}\left(\mathbf{T}^{n}\right)\right)$ defined by $\mu(A)=\varphi(A), A \in \mathcal{P}\left(\mathbf{T}^{n}\right)$. Then clearly $\mu(R(C))=1$. For any proper subtorus $D$ of $C, \mu_{s}(R(D))=0$ for all $s$ and hence $\mu(R(D))=0$. Finally, it is obvious that if $A=\left\{x \mid x^{j} \in C\right.$ for $\left.j \leq s\right\}$, then $\mu_{t}(A) \leq N_{s} / N_{t}$, in the above notation, so $\mu(A)=0$.

3. Absolutely continuous invariant measures. We now proceed to prove Theorem 1. In view of Corollary 2.5 we need to prove it only under the additional hypothesis that $\mu$ be absolutely continuous with respect to $m$. The following lemma shows we may further assume that the $H$-action on $\mathbf{T}^{n}$ is ergodic, or, equivalently, that there is no countably additive $H$-invariant absolutely continuous measure on $\mathbf{T}^{n}$ other than $m$.

3.1 LEMmA. Let $H$ be a subgroup of $\operatorname{GL}(n, \mathbf{Z})$. If the $H$-action on $\mathbf{T}^{n}$ is not ergodic (with respect to $m$ ) there exists a proper closed connected $H$-invariant subgroup $C$ such that $H\left(\mathbf{T}^{n} / C\right)$ is finite; in particular, it is amenable.

PROOF. It is well known that the $H$-action on $\mathbf{T}^{n}$ is ergodic if and only if under the adjoint action of $H$ on $\mathbf{Z}^{n}$ there are no finite orbits except for that of the trivial character 0 . (See the proof in $[\mathbf{7}]$ for a single automorphism for an idea of the general proof.) Thus under the hypothesis of the lemma,

$$
X=\left\{\gamma \in \mathbf{Z}^{n} \mid H \text {-orbit of } \gamma \text { is finite }\right\}
$$

is a nontrivial $H$-invariant subgroup. Further, $X$ is clearly a finitely generated abelian group and, hence, every orbit of the $H$-action being finite implies that a subgroup $H_{0}$ of finite index in $H$ fixes $X$ pointwise. Thus if $C=\operatorname{ann} X=\{x \in$ $\mathbf{T}^{n} \mid \gamma(x)=1$ for all $\left.\gamma \in X\right\}$ then $C$ is a proper closed $H$-invariant subgroup of $\mathbf{T}^{n}$ and $H\left(\mathbf{T}^{n} / C\right) \simeq H / H_{0}$ is finite. Also it is evident from the definition that $\mathbf{Z}^{n} / X$ has no torsion elements, so, by duality $C$ is connected, as required.

Now suppose $H$ is a subgroup of $\operatorname{GL}(n, \mathbf{Z})$ acting ergodically on $\mathbf{T}^{n}$ and let $\mu$ be an $H$-invariant finitely additive probability measure on $\mathbf{T}^{n}$ absolutely continuous with respect to $m$, but $\mu \neq m$. In this case the map $f \mapsto \int f d \mu$, for all bounded Lebesgue measurable functions, induces an $H$-invariant mean $\psi$ on $L^{\infty}\left(\mathbf{T}^{n}, \mathcal{L}, m\right)$ different from the Haar integral. As noted in $\S 1$ we identify $\mathbf{Z}^{n}$ as the character group of $\mathbf{T}^{n}$ and the (contragradient) $\mathrm{GL}(n, \mathbf{Z})$-action as the adjoint (left) action on the character group. Then given a mean $\psi$ as above, by a theorem of Rosenblatt (cf. [4. Theorem 1.4 and Proposition 3.2]) there exists an $H$-invariant mean $\varphi$ on $B\left(\mathbf{Z}^{n}\right)$ (the space of all bounded functions) such that $\varphi(\{0\})=0$.

Let $W$ be a minimal $H$-invariant subspace of $\mathbf{R}^{n}$ with the property $\varphi\left(W \cap \mathbf{Z}^{n}\right)=$ 1. Let $C \subset \mathbf{T}^{n}$ be the annihilator subgroup of $W \cap \mathbf{Z}^{n}$. Then $C$ is a closed connected $H$-invariant subgroup of $\mathbf{T}^{n}$ and $W \cap \mathbf{Z}^{n}$ is the character group of $\mathbf{T}^{n} / C$. Since $0=\varphi(\{0\})<\varphi\left(W \cap \mathbf{Z}^{n}\right)=1, W \cap \mathbf{Z}^{n} \neq\{0\}$ and hence, by duality, $C$ is a proper subgroup of $\mathbf{T}^{n}$. We claim that assertion (i) is satisfied for this subgroup $C$.

By changing notation we may view $\mathbf{T}^{n} / C$ itself as the original torus $\mathbf{T}^{n}$ and $H\left(\mathbf{T}^{n} / C\right)$ as the original subgroup $H$ (which we would now like to prove to be amenable). This entails replacing $W \cap \mathbf{Z}^{n}$ as above by $\mathbf{Z}^{n}$, and we get an $H$ invariant mean on $B\left(\mathbf{Z}^{n}\right)$ such that $\varphi(\{0\})=0$ and, further, $\varphi\left(U \cap \mathbf{Z}^{n}\right)<1$ for 
any proper $H$-invariant subspace $U$ of $\mathbf{R}^{n}$. Suppose $H$ is not amenable. Let $N$ be the subgroup generated by $\{S \in \mathcal{M}(H)\}$ (cf. $\S 1$ ); then $N$ is a nontrivial normal subgroup of $H$. Also by Proposition $2.2 \varphi(E(N))=1$, where $E(N)$ is the subgroup of $\mathbf{Z}^{n}$ consisting of vectors fixed by $N$ (under the contragradient action). Since $N$ is normal in $H, E(N)$ is $H$-invariant. Further, evidently $E(N)$ is of the form $W \cap \mathbf{Z}^{n}$. where $W$ is an $H$-invariant subspace of $\mathbf{R}^{n}$. Since $\varphi\left(U \cap \mathbf{Z}^{n}\right)<1$ for any proper $H$-invariant subspace, we get $E(N)=\mathbf{Z}^{n}$. But since $N$ is nontrivial, this is not possible; viz. every vector cannot be fixed by $N$. Hence $H$ is amenable.

4. Invariant measures for amenable subgroups. In this section we discuss invariant measures of actions of amenable subgroups of $\operatorname{GL}(n, \mathbf{Z})$ and give a proof of Theorem 4.

4.1 Proposition. Let $H$ be an amenable subgroup of $\mathrm{GL}(n, \mathbf{Z}), n>1$, and consider the $H$-action on $\mathbf{T}^{n}$. Then there exists an $H$-invariant finitely additive probability measure $\mu$ on $\left(\mathbf{T}^{n}, \mathcal{L}\right)$ such that $\mu$ is absolutely continuous with respect to (the normalised Haar measure) $m$, but $\mu \neq m$.

Proof. Let $\mathcal{N}=\left\{E \in \mathcal{P}\left(\mathbf{T}^{n}\right) \mid\right.$ there exists a Borel subset $B$ such that $E \subset B$ and $m(B)=0$. Let $A$ be a closed nowhere dense subset of $\mathbf{T}^{n}$ such that $m(A)>0$. Let $A$ be the smallest $H$-invariant algebra containing $\mathcal{N}$ and all subsets of $A$. Let

$$
\begin{aligned}
C=\left\{C=E \cup F \mid E \in \mathcal{N} \text { and there exist } h_{1}, h_{2}, \ldots, h_{k} \text { in } H\right. \\
\text { such that } \left.F \subset h_{1} A \cup h_{2} A \cup \cdots \cup h_{k} A\right\} .
\end{aligned}
$$

It is easy to verify that $A$ consists precisely of sets from $C$ and their complements in $\mathbf{T}^{n}$. We also note that if $C \in \mathcal{C}$, then $\mathbf{T}^{n}-C$ does not belong to $C$; otherwise therc would exist finitely many elements $h_{1}, h_{2}, \ldots, h_{r}$ in $H$ such that $m\left(\mathbf{T}^{n}-\bigcup_{1}^{r} h_{i} A\right)=$ 0 , which is impossible since $A$ is a closed nowhere dense set. Hence on $A$ we can define a finitely additive probability measure $\mu_{0}$ by setting $\mu_{0}(A)=0$ if $A \in C$ and $\mu_{0}(A)=1$ if $\mathbf{T}^{n}-C \in C$. Notice that $\mu_{0}$ is $H$-invariant. Since $H$ is amenable Theorem 5.1 in $[3]$ implies that $\mu_{0}$ extends to an $H$-invariant finitely additive probability measure on $\left(\mathbf{T}^{n}, \mathcal{P}\left(\mathbf{T}^{n}\right)\right)$. Let $\mu$ be its restriction to $\left(\mathbf{T}^{n}, \mathcal{L}\right)$. Since $\mu$ extends $\mu_{0}$ and $\mu_{0}(E)=0$ for all $E \in \mathcal{N}$, we conclude that $\mu$ is absolutely continuous with respect to $m$. On the other hand $\mu \neq m$ since $\mu(A)=0<m(A)$. Thus $\mu$ has the desired properties.

Proposition 4.1 can also be proved by constructing an 'asymptotically invariant sequence of sets' and then using the correspondence as in [4]. The author had originally followed this approach. The author is thankful to S. Wagon and J. Mycielski for suggesting the present proof, which is shorter and more direct.

PROOF OF THEOREM 4. In view of Theorem 2.7 we need to prove Theorem 4 only under condition (i); that is, $H\left(\mathbf{T}^{n} / C\right)$ amenable, with $H$ and $C$ as in the statement of the theorem. By Proposition 4.1 there exists an $H\left(\mathbf{T}^{n} / C\right)$-invariant finitely additive probability measure $\mu_{C}$ on $\mathbf{T}^{n} / C$ defined for all Lebesgue measurable sets of $\mathbf{T}^{n} / C$ and absolutely continuous with respect to, but different from, the normalised Haar measure on $\mathbf{T}^{n} / C$. Let $\lambda_{C}$ denote the normalised Haar measure on $C$. We define a finitely additive probability measure $\mu$ on $\left(\mathbf{T}^{n}, \mathcal{L}\right)$ as follows: Let $E \in \mathcal{L}$ and let $\chi_{E}$ be the characteristic function of $E$. Then there exists a Lebesgue measurable set $N$ of $\mathbf{T}^{n} / C$ such that $\mu_{C}(N)=0$ and, for all $x \in \mathbf{T}^{n}$ such 
that $x C \notin N, \chi_{E}(x t)$ is a measurable function of $t \in C$. Further, putting

$$
\psi_{E}(x C)= \begin{cases}\int_{0} \chi_{E}(x t) d \lambda_{C}(t) & \text { if } x C \notin N \\ \text { otherwise }\end{cases}
$$

defines a Lebesgue measurable function $\psi_{E}$ on $\mathbf{T}^{n} / C$. Put $\mu(E)=\int \psi_{E} d \mu_{C}$. Using the fact that $\mu_{C}$ is absolutely continuous, it is easy to see that $\mu$ is a finitely additive probability measure. It is also straightforward to check that $\mu$ is $H$-invariant and absolutely continuous with respect to $m$. Finally, $\mu \neq m$ since the two do not even coincide on all $C$-invariant sets.

5. Conclusions and comments. The simplest application of Theorem 1 arises when there is no nontrivial proper subtorus $C$ invariant under $H$, that is, when $H$ acts irreducibly. In this case we get Corollary 2. On the other hand if $H$ is a subgroup of $\operatorname{GL}(n, \mathbf{Z})$ such that the 'adjoint' or, equivalently, the contragradient action on $\mathbf{Z}^{n}-(0)$ is free, then clearly for any proper $H$-invariant subtorus $C$, $H\left(\mathbf{T}^{n} / C\right)$ is isomorphic to $H$. This immediately leads to Corollary 3 .

Consider next the action of $\operatorname{SL}(2, \mathbf{Z})$ on $\mathbf{T}^{n}, n \geq 2$, defined as follows. If $n$ is even, $n=2 p$ say, consider $\mathbf{T}^{n}$ as $\mathbf{T}^{2} \times \mathbf{T}^{2} \times \cdots \times \mathbf{T}^{2}$ ( $p$ copies) and let $\operatorname{SL}(2, \mathbf{Z})$ act componentwise, where on each component we have the usual action. On $\mathbf{T}^{3}$ we have an $\operatorname{SL}(2, \mathbf{Z})$-action defined as follows: Identify $\mathbf{R}^{3}$ with the space of $2 \times 2$ real matrices with trace 0 and $\mathbf{Z}^{3}$ with the subgroup consisting of integral matrices with trace 0 . The action of $\operatorname{SL}(2, \mathbf{Z})$ on $2 \times 2$ matrices of trace 0 defined by $A(X)=$ $A X A^{-1}$, where $A \in \mathrm{SL}(2, \mathbf{Z}), X$ of trace 0 , gives an $\mathrm{SL}(2, \mathbf{Z})$-action on $\mathbf{R}^{3}$ leaving $\mathbf{Z}^{3}$ invariant. Thus we get a $\mathrm{SL}(2, \mathbf{Z})$-action on $\mathbf{T}^{3}$. For any odd $n>3$, say $n=2 p+3$, we get an SL $(2, \mathbf{Z})$-action on $\mathbf{T}^{n}$ by considering $\mathbf{T}^{n}$ as $\mathbf{T}^{2 p} \times \mathbf{T}^{3}$ and letting $\mathrm{SL}(2, \mathbf{Z})$ act componentwise, where on the components we have actions as above.

It may be noted that the adjoint action of the above $\operatorname{SL}(2, \mathbf{Z})$-action on $\mathbf{T}^{n}$ is not free. However we have the following.

5.1 Proposition. Consider the $\mathrm{SL}(2, \mathbf{Z})$-action on $\mathbf{T}^{n}, n \geq 2$, as defined above. Let $\mu$ be an $\mathrm{SL}(2, \mathbf{Z})$-invariant finitely additive probability measure defined on $\left(\mathbf{T}^{n}, \mathcal{L}\right)$. Then either $\mu=m$ (the normalised Haar measure) or $\mu(R)>0$, where $R$ is the set of elements of finite order in $\mathbf{T}^{n}$.

PROOF. Any proper invariant subtorus $C$ arises only by setting a certain number of components to be the identity. For any such nontrivial $C, H(C)$ and $H\left(\mathbf{T}^{n} / C\right)$ are isomorphic to either $\mathrm{SL}(2, \mathbf{Z})$ or $\mathrm{SL}(2, \mathbf{Z}) /\{ \pm I\}$, where $I$ is the identity matrix; therefore they are never amenable $(\mathrm{cf} .[\mathbf{1}])$. The proposition therefore follows immediately from Theorem 1.

More generally if $H$ is a subgroup of $\operatorname{GL}(n, \mathbf{Z})$ whose action on $\mathbf{T}^{n}$ is completely reducible (that is, there exist $H$-invariant subtori $C_{1}, \ldots, C_{k}$ such that $\mathbf{T}^{n}=C_{1} C_{2} \cdots C_{k}$ and the $H$-action on each $C_{i}$ is irreducible), then a similar analysis is possible. The analogous conclusion holds if for each $i$ the restrictions of elements of $H$ to $C_{i}$ constitute a nonamenable subgroup. 


\section{REFERENCES}

1. F. P. Greenleef, Invariant means on topological groups, Van Nostrand, New York, 1969.

2. A. del Junco and J. Rosenblatt, Counterexamples in ergodic theory and number theory, Math. Ann. 245 (1979), 185-197.

3. J. Mycielski, Finitely additive invariant measures. I, Colloq. Math. 42 (1979), 309-318.

4. J. Rosenblatt, Uniqueness of invariant means for measure-preserving transformations, Trans. Amer. Math. Soc. 265 (1981), 623-636.

5. J. Tits, Free subgroups of linear groups, J. Algebra 20 (1972), 250-270.

6. S. Wagon, Invariance properties of finitely additive measures on $R^{n}$, Illinois J. Math. 25 (1981), 74-86.

7. P. Walters, Ergodic theory -introductory lectures, Lecture Notes in Math., vol. 458, Springer, New York, 1975.

SChool of Mathematics, TAta institute of Fundamental Research, homi BHABHA ROAD, BOMBAY 400005 INDIA 